\title{
CT Pulmonary Angiography Findings in Children with Congenital Heart Disease in a Tertiary Care Hospital
}

\author{
Nusrat Ghafoor, ${ }^{1}$ Nawshin Siraj ${ }^{2}$
}

\begin{abstract}
Background \& objective: Computed tomography (CT) is claimed to accurately diagnose many forms of congenital cardiovascular disease in children, particularly in the diagnosis of extracardiac vascular anomalies. The present study is intended to describe the role of CT in the evaluation of congenital cardiovascular disease in children.

Methods: The study included children with congenital heart disease (CHD) who underwent CT pulmonary angiography in the Cardiac Imaging Department of Ibrahim Cardiac Hospital \& Research Institute, Dhaka between 2015-2017 for investigations of various congenital heart diseases. CT scan was done on Multidetector scanner from Siemens or Philips. Routine preprocedure was done as per protocol of the CT angiography study. Breath holding technique was explained to the older patients who were able to follow breath holding instructions. Plain CT scans of thorax followed by early arterial phases were obtained after negative test dose of the non-ionic contrast by injecting 12 to $40 \mathrm{ml}$ of intravenous contrast media (Iopamidol $350 \mathrm{mg}$ ) through antecubital vein by pressure injector at a rate of $1.5 \mathrm{ml} /$ second Scan was obtained 6 seconds after starting of the intravenous contrast.

Results: The mean age of the patients was $5.3 \pm 0.5$ years. Over $60 \%$ of the children were male giving a male to female ratio of roughly $3: 2$. Majority (92\%) of children had levocardia with aorta being predominantly originated from the left ventricle (95\%). Nearly two-thirds (65\%) of the aortas had $50 \%$ overriding, 33\% had $>50 \%$ overriding. Over three-quarters (76\%) had left-sided arch and $21 \%$ had right-sided arch. Majority $(87 \%)$ of pulmonary arteries originated from the right ventricle: $50 \%$ were of normal size and $44 \%$ were narrower than normal. In a few cases both right and left pulmonary artery were absent. In $90 \%$ cases right and left pulmonary arteries and veins were confluent. Nearly two-thirds (64\%) of the pulmonary arteries were stenosed and $5 \%$ did not have any pulmonary valves. Presence of collaterals was commendably high (42\%). Seventy percent of the patients had Tetralogy of Fallot (TOF) and over one-quarter (26\%) had Patent Ductus Arteriosus (PDA). Interatrial septal defect was found in $21 \%$ and intervetricular septal defect in $79 \%$ cases. Increased and decreased vascularity of the lungs were found in 13 and $15 \%$ of the cases respectively. Other cardiac anomalies were negligible.
\end{abstract}

Conclusion: The findings of the study suggest that the CT pulmonary angiography in the evaluation of CHD has a number of merits and could be done in the diagnosis of pulmonary vascular structures particularly the extra cardiac vascular anomalies.

Key words: CT Pulmonary Angiography, cardiovascular anomalies, children, congenital heart disease etc.

\section{INTRODUCTION:}

Computed tomography (CT), including CT angiography (CTA), is important in the evaluation of pediatric congenital heart disease (CHD). It can be used for accurate depiction of complex cardiovascular anatomic features, particularly in the assessment of extracardiac systemic and pulmonary arterial and venous structures. It is, therefore, important for radiologists to have extensive knowledge of cardiovascular anatomy, physiology, and surgical techniques. ${ }^{1}$ Although echocardiography and catheter-directed cardiac

\footnotetext{
Authors' information:

${ }^{1}$ Dr. Nusrat Ghafoor, Associate Consultant, Department of Radiology \& Imaging, ICHRI, Dhaka

${ }^{2}$ Dr. Nawshin Siraj, Senior Consultant \& Head of the Department of Radiology \& Imaging, ICHRI, Dhaka
}

Correspondence: Dr. Nusrat Ghafoor, Cell Phone: +880 1711316595 Email: ghafoornusrat@yahoo.com 
angiography are generally accepted as the primary imaging techniques for evaluation of CHD, CT and MRI are important complementary diagnostic tools. In addition to being highly operator dependent, ${ }^{2}$ echocardiography may not be sufficient for evaluating extracardiac structures, such as the pulmonary arteries, pulmonary veins, and the aortic arch and great vessels due to acoustic window limitations. ${ }^{2,3}$

Compared with $\mathrm{CT}$, cardiac catheterization has a higher complication rate owing to its invasive nature..$^{4,5}$ The latter frequently requires a larger volume of intravascular contrast material and general anesthesia. ${ }^{4}$ It often imparts greater radiation dose to the patient when appropriate pediatric CT protocols are used. ${ }^{1}$ CT and MRI can be used as problem-solving tools, especially in the evaluation of preoperative and postoperative cardiovascular anatomical assessment and certain postoperative complications. Although MRI does not cause ionizing radiation and can be used for functional assessment of cardiovascular structures and for dynamic multiphasic contrast-enhanced imaging, it has several disadvantages compared with $\mathrm{CT}$, which among others, include poorer spatial resolution; ${ }^{2,6}$ the presence of imagedegrading artifacts from implanted metal, such as intravascular stents and embolization coils; ${ }^{7}$ higher cost; ${ }^{2,8}$ limited availability; ${ }^{2,6}$ and increased need for general anesthesia in younger children. ${ }^{6}$

CT offers excellent spatial resolution and relatively unrestricted access in much shorter acquisition times than MRI. ${ }^{9}$ It is well suited for imaging the epicardial coronary arteries and their relations to adjacent structures or conduits. New generation Multidetector Computed Tomography (MDCT) has important roles in overcoming these limitations. CT angiography is fast method to evaluate vascular structural anomalies by fast image acquisition times and their capacity to obtain volumetric data. For the complete evaluation of CHD high and spatial resolution is necessary which is possible with $\mathrm{CT}$ imaging. ${ }^{10}$ The present study is intended to illustrate and describe the CT findings in patients with CHD (extra cardiac abnormalities, cardiac abnormalities, connection problems), and the advantages \& disadvantages of $\mathrm{CT}$ in this setting.

\section{METHODS:}

The study included children with congenital heart disease who underwent CT pulmonary angiography in the Cardiac Imaging Department of Ibrahim Cardiac Hospital \& Research Institute, Dhaka between 2015-2017 for investigations of various congenital heart diseases. CT scan was done on Multidetector scanner from Siemens or Philips. Written informed consent was obtained from the parents/guardians of the children with CHD. Routine preprocedure was done as per the protocol of the CT angiography study. Breath holding technique was explained to the older patients who was able to follow breath holding instructions. Plain CT scans of thorax followed by early arterial phases was obtained after negative test dose of the non-ionic contrast by injecting 12 to $40 \mathrm{ml}$ of intravenous contrast media (Iopamidol $350 \mathrm{mg}$ ) through antecubital vein by pressure injector at a rate of $1.5 \mathrm{ml} / \mathrm{sec}$. Scan was obtained 6 second after starting of the intravenous contrast. Scan parameters were as follows: Volumetric data were obtained from the vessels in axial plane and were reconstructed in saggital and coronal plane. Slice thickness was $1 \mathrm{~mm}$. Collimation: 0.6 mm. Pitch:-1.5, MAS=200, Kvp120.

\section{RESULTS:}

The mean age of the patients was $5.3 \pm 0.5$ years. Over $60 \%$ of the children were male giving a male to female ratio of roughly 3:2 (Table I). Majority $(92 \%)$ of children had levocardia with aorta being predominantly originated from the left ventricle $(95 \%)$. Nearly two-thirds (65\%) of the aortas had $50 \%$ overriding, $33 \%$ had $>50 \%$ overriding. Over three-quarters $(76 \%)$ had left-sided arch and $21 \%$ right-sided arch. Majority (87\%) of pulmonary arteries originated from the right ventricle- $50 \%$ were of normal size and $44 \%$ were 
narrower than normal. Sixty percent of the right pulmonary arteries and $56 \%$ of the left pulmonary arteries were narrower. In a few cases both right and left pulmonary arteries were absent. In 90\% cases right and left pulmonary arteries and veins were confluent. Nearly two-thirds (64\%) of the pulmonary arteries were stenosed and $5 \%$ did not have any pulmonary valves (Table II).

One-quarter of the right atrium, $8 \%$ of left atrium, $23 \%$ of right ventricle and $10 \%$ of left ventricle were dilated. Small left atrium was found in $14 \%$ cases. However, single atrium and ventricle were rare. Presence of collaterals was commendably high (42\%). Double superior vena cava and dilated coronary sinus were found in $11 \%$ and $12 \%$ cases respectively. Over one-quarter ( $26 \%)$ children had Patent Ductus Arteriosus (PDA). Interatrial septal defect was found in $21 \%$ and intervetricular septal defect in $79 \%$ cases. Increased and decreased vascularity of the lungs were found in 13 and $15 \%$ of the cases respectively (Table III). CT diagnosis revealed that $70 \%$ of the patients had Tetralogy of Fallot (TOF) and $26 \%$ had PDA. Total anomalous pulmonary venous connection (TAPVC) and partial anomalous pulmonary venous connection (PAPVC) were 9 and $16 \%$ respectively, while coarctation of aorta and single ventricle were 3 and $2 \%$ cases respectively. Other anomalies were AP window $(2 \%)$, single atrium (1\%), Anomalous origin of the left coronary artery from the pulmonary artery (ALCAPA) (2\%), Atrial Septal Defect (ASD) with PLSVC (2\%), Double outlet right ventricle (DORV) $(2 \%)$, large VSD (1\%) (Table IV).

\begin{tabular}{|c|c|c|c|}
\hline $\begin{array}{l}\text { Demographic } \\
\text { variables }\end{array}$ & Frequency & Percentage & $\begin{array}{c}\text { Mean } \pm \text { SEM } \\
\quad \text { (range) }\end{array}$ \\
\hline Age (yrs) & - & - & $5.3 \pm 0.5(0.1-24)$ \\
\hline \multicolumn{4}{|l|}{ Sex } \\
\hline Male & 62 & 62.0 & - \\
\hline Female & 38 & 38.0 & - \\
\hline
\end{tabular}

TABLE II. Features of aorta and pulmonary arteries

\begin{tabular}{lcc|}
$\begin{array}{l}\text { Features of aorta \& } \\
\text { pulmonary artery }\end{array}$ & Frequency & Percentage \\
$\begin{array}{l}\text { Cardiac position } \\
\text { Laevocardia }\end{array}$ & 92 & 92.0 \\
Dextrocardia & 7 & 7.0 \\
Mesocardia & 1 & 1.0 \\
Origin of aorta & & \\
LV & 95 & 95.0 \\
RV & 5 & 5.0 \\
Overriding of aorta (\%) & & \\
50\% & 65 & 65.0 \\
$>50 \%$ & 33 & 33.0 \\
No overriding & 2 & 2.0 \\
Arch & & 76.0 \\
Left-sided & 76 & 21.0 \\
Right-sided & 21 & 3.0 \\
Absent & 3 &
\end{tabular}

Origin PA

LV

8.0

RV

87.0

Single ventricular

5.0

Size of PA

Normal

50.0

Narrow

44.0

Absent

6.0

Size of RPA

Normal

38.0

Narrow

60.0

Absent

60

2.0

Size of LPA

Normal

42.0

Narrow

56.0

Absent

2.0

Confluence of RPA \& LPA

90.0

Pulmonary veins

Normal

83.0

Abnormal

17.0

Pulmonary valve

Normal

31.0

Stenosed

64.0

Absent 


\begin{tabular}{|c|c|c|c|c|c|}
\hline \multicolumn{3}{|c|}{ TABLE III. Anomalies of cardiac chambers } & \multicolumn{3}{|c|}{$\begin{array}{l}\text { TABLE IV. Distribution of patients by diagnosis of congenital } \\
\text { heart diseases }\end{array}$} \\
\hline Cardiac chamber & Frequency & Percentage & СТ Diagnosis & equency & Percentage \\
\hline \multicolumn{3}{|l|}{ Right atrium } & TOF & 70 & 70.0 \\
\hline Normal & 74 & 74.0 & TAPVC & 9 & 9.0 \\
\hline Dilated & 25 & 25.0 & PAPVC & 4 & 4.0 \\
\hline Single & 1 & 1.0 & PDA & 26 & 26.0 \\
\hline \multicolumn{3}{|l|}{ Left atrium } & \multirow{3}{*}{\multicolumn{2}{|c|}{$\begin{array}{l}\text { Coarctation of the aorta } \\
\text { Single ventricle } \\
\text { Others }\end{array}$}} & \multirow{3}{*}{$\begin{array}{l}3.0 \\
2.0\end{array}$} \\
\hline $\begin{array}{l}\text { Normal } \\
\text { Dilated }\end{array}$ & $\begin{array}{l}77 \\
8\end{array}$ & $\begin{array}{c}77.0 \\
8.0\end{array}$ & & & \\
\hline Small & 14 & 14.0 & & & \\
\hline Single & 1 & 1.0 & \multirow{2}{*}{$\begin{array}{l}\text { AP window } \\
\text { Single atrium }\end{array}$} & 2 & 2.0 \\
\hline \multicolumn{3}{|l|}{ Right ventricle } & & 1 & 1.0 \\
\hline Normal & 72 & 72.0 & ALCAPA & 2 & 2.0 \\
\hline Dilated & 23 & 23.0 & \multirow{2}{*}{$\begin{array}{l}\text { Long VSD with pulmonary artery atresia } \\
\text { ASD with PLSVC }\end{array}$} & 1 & 1.0 \\
\hline Small & 3 & 3.0 & & 2 & 2.0 \\
\hline Single & 2 & 2.0 & DORV & 2 & 2.0 \\
\hline Left ventricle & & & Large VSD & 1 & 1.0 \\
\hline
\end{tabular}

\begin{tabular}{|c|c|c|}
\hline Normal & 83 & 83.0 \\
\hline Dilated & 10 & 10.0 \\
\hline
\end{tabular}

Small $\quad 5.0$

Single 2.0

Presence of collaterals $\quad 42 \quad 42.0$

SVC

$\begin{array}{lll}\text { Single } & 89 & 89.0\end{array}$

Double $\quad 11 \quad 11.0$

Coronary sinus (CS)

Normal $\quad 86 \quad 86.0$

$\begin{array}{lll}\text { Dilated } & 12 & 12.0\end{array}$

$\begin{array}{lll}\text { Not seen } & 2 & 2.0\end{array}$

$\begin{array}{lll}\text { PDA } & 26 & 26.0\end{array}$

Interatrial septum

$\begin{array}{lll}\text { Intact } & 78 & 78.0\end{array}$

Defect $21 \quad 21.0$

$\begin{array}{lll}\text { Absent } & 1 & 1.0\end{array}$

Inter ventricular septum

$\begin{array}{lcc}\text { Intact } & 19 & 19.0 \\ \text { Defect } & 79 & 79.0 \\ \text { Absent } & 2 & 2.0 \\ \text { Lungs findings } & & \\ \text { Normal vascularity } & 63 & 63.0 \\ \text { Increase vascularity } & 13 & 13.0 \\ \text { Decrease vascularity } & 15 & 15.0 \\ \text { Infection } & 9 & 9.0\end{array}$

In the present study, pulmonary arteries were found to be predominantly originated from the right ventricle $(87 \%)$. Half of them was of normal size and an almost half (44\%) was narrower than normal. In a few cases both right and left pulmonary arteries were absent. In majority of the cases right and left pulmonary arteries and veins were confluent. Nearly two-thirds (64\%) of the pulmonary arteries were stenosed with absence of valve in few cases. Presence of collaterals was indeed high.

Compared with CT of the past, 16-MDCT and newer scanners yield images with better temporal and spatial resolution, greater anatomic coverage per rotation, more consistent enhancement with a lesser volume of intravascular contrast material, and higher-quality 2D reformation and 3D reconstruction owing to acquisition of an isotropic data set. ${ }^{11,12}$ Rapid imaging could be done with these CT scanners. Although the role of CT in the evaluation of pediatric CHD is continually being redefined and expanded, there are several generally accepted clinical indications for which the benefits of imaging outweigh the risks. First, $\mathrm{CT}$ can be used to evaluate patients with $\mathrm{CHD}$ 
suspected on the basis of echocardiographic findings for which further imaging is needed to characterize extracardiac anomalies before intervention, for the pulmonary arteries, pulmonary veins, \& aortic arch and great vessels, which are often inadequately characterized at echocardiography. ${ }^{3}$ Second, CT can be used in the postoperative evaluation of $\mathrm{CHD}$ patients who may have a variety of suspected treatmentrelated complications. ${ }^{7}$ Third, as CT facilitates rapid and comprehensive assessment of thoracic cardiovascular structures, the lungs, and potential spaces, it can be used in the preoperative and postoperative evaluation of CHD patients whose condition abruptly deteriorates. ${ }^{7}$ In this situation, CT quickly displays evidence of a variety of CHD-related complications and numerous other medical conditions, such as pulmonary embolism, pneumonia, pleural and pericardial effusion, and pneumothorax. $^{7}$

Finally, CT could be useful before a reoperation to assess altered anatomic features related to previous surgery. ${ }^{7}$ CT can be considered as the first-line imaging technique for a few clinical indications. Examples are evaluation of suspected vascular ring or sling, suspected aortopulmonary collateral arteries in patients with severe right ventricular outflow tract obstruction, \& individuals with implanted pacemakers and metal surgical hardware where MRI is contraindicated. ${ }^{6,7}$ The role of CT in the evaluation of intracardiac anomalies has been limited, although the situation is changing owing to advances in MDCT scanners and ECG-gating techniques. In a small study, Lee et al. $^{13}{ }^{13}$, in which they used a 40-MDCT scanner (without ECG gating), detected 53 of 54 intracardiac \& extracardiac anomalies in neonates with complex congenital heart disease. The pediatric thoracic CTA usually requires mild or no sedation. Unlike the practice for MRI and cardiac catheterization, general anesthesia is infrequently needed for CTA because quiet breathing rarely causes appreciable artifacts. ${ }^{3,8,14,15}$

The findings of the present study suggest that CT pulmonary angiography could dependably diagnose $\mathrm{CHD}$ in children. The accumulating evidences from other studies as well suggest that the CT pulmonary angiography in the evaluation of $\mathrm{CHD}$ has a number of merits and could be done in the diagnosis of pulmonary vascular structures with fair degree of accuracy.

\section{REFERENCES:}

1. Gaca AM, Jaggers JJ, Dudley LT, Bisset GS 3rd. Repair of congenital heart disease: a primer. Part 1. Radiology 2008;247:617-631.

2. Tsai IC, Chen MC, Jan SL Wang CC, Fu YC, Lin PC, Lee T. Neonatal cardiac multidetector row CT: why and how we do it. Pediatr Radiol 2008;38:438-451.

3. Leschka S, Oechslin E, Husmann L, Desbiolles $L$, Marincek B, , Genoni M, Pretre R, Jenni R, Wildermuth $\mathrm{S}$, Alkadhi $\mathrm{H}$. Pre and postoperative evaluation of congenital heart disease in children and adults with 64-section CT. Radio Graphics 2007;27:829-846.

4. Hollingsworth $\mathrm{CL}$, Yoshizumi $T$, Frush $\mathrm{DP}$, Chan $\mathrm{FP}$ Toncheva G, Nguyen G, Lowry CR, Hurwitz LM. Pediatric cardiac-gated $\mathrm{CT}$ angiography: assessment of radiation dose. AJR 2007;189:12-18.

5. Mehta R, Lee KJ, Chaturvedi R, Benson L. Complications of pediatric cardiac catheterization: a review in the current era. Catheter Cardiovasc Interv 2008;72: 278-85.

6. Taylor AM. Cardiac imaging: MR or CT? Which to use when. Pediatr Radiol 2008;38(suppl 3):S433-S438.

7. Bean MJ, Pannu H, Fishman EK. Three-dimensional computed tomographic imaging of complex congenital cardiovascular abnormalities. J Comput Assist Tomogr 2005;29:721-24.

8. Frush DP, Herlong JR. Pediatric thoracic CT angiography. Pediatr Radiol 2005;35:11-25.

9. Cook SC, Raman SV. Multidetector computed tomography in the adolescent and young adult with congenital heart disease. J Cardiovasc Comput Tomogr 2008;2: 36-49.

10. Vaishnav KU, Bhatt CJ, Bolla SD, Shah DS. Role of pulmonary angiography in congenital heart diseases among children. 2013;3(4):332-37. 
11. Siegel MJ. Multiplanar and three-dimensional multi-detector row CT of thoracic vessels and airways in the pediatric population. Radiology 2003;229:641-50.

12. Spevak PJ, Johnson PT, Fishman EK. Surgically corrected congenital heart disease: utility of 64- MDCT. AJR 2008;191:854-61.

13. Lee $\mathrm{T}$, Tsai IC, Fu YC, Jan SL, Wang CC, Chang Y, Chen MC. Using multidetectorrow $\mathrm{CT}$ in neonates with complex congenital heart disease to replace diagnostic cardiac catheterization for anatomical investigation: initial experiences in technical and clinical feasibility. Pediatr Radiol 2006;36:1273-82.
14. Hopkins KL, Patrick LE, Simoneaux SF, Bank ER, Parks WJ, Smith SS. Pediatric great vessel anomalies: initial clinical experience with spiral CT angiography. Radiology $1996 ; 200: 811-15$

15. Lee KH, Yoon CS, Choe KO, Choe KO, Kim MJ, Lee $\mathrm{HM}$, Yoon HK, Kim B. Use of imaging for assessing anatomical relationships of tracheobronchial anomalies associated with left pulmonary artery sling. Pediatr Radiol 2001;31:269-78. 